\title{
Melanismus bei Lepidopteren als Mutation und individuelle Variation.
}

Von M. Willy Gerschler, Leipzig, Zoologisches Institut.

Mit 1 Textfigur und 2 Tafeln.

(Eingegangen am 7. Februar 1913.)

In den Jahren 1910-12 waren im hiesigen Zoologischen Institut auf Veranlassung des Herrn Geheimrat CHus eine Reihe von Kreuzungsexperimenten ausgeführt worden, deren Bearbeitung und Deutung bislang ausstand. Es handelt sich um den Birkenspanner, Amphidasys betularizs L. und seine schwarze Aberration doubledayaria Mill. Gleich an dieser Stelle sei Herrn Geheimrat CHuN für seine Güte gedankt, die es mir erlaubt, den Fall im folgenden zu verwerten. Bei den großen Zahlen des ersten Versuchs und dem positiven Ausfall der sich anschließenden, die die Probe auf das Exempel darstellen, läßt sich ihm theoretisch einige Bedeutung beilegen. Damit aber war die sehr erwünschte Möglichkeit gegeben, frühere Experimente, die in der Literatur vorliegen, kritisch nachzuprüfen. Doch wurde dabei kein einseitiger MaBstab angelegt. Vielmehr stellte es sich heraus, daß die zahlreichen Fortschritte, die die Vererbungslehre in den letzten Jahren gemacht hat, auch hier neue Perspektiven eröffneten.

Wenn sich der neu mitzuteilende Amphidasys-Fall durchaus auf gleicher Linie wie STANDFuss' ausgezeichnete Versuche mit Aglia tau und ihren Aberrationen bewegt, so kommen wir jener Gesetzmäßigkeit immer näher, die auch für die Melanomutationen der Lepidopteren keine Besonderheiten in der Vexerbungsweise annimmt, sondern sie ausnahmslos den Mendelistischen Phänomenen eimreiht.

Die vererbungstheoretische Interpretation des Melanismus bei Lepidopteren hat im Laufe der Zeit manche Wandlungen durchgemacht. Die ersten und klassischen Experimente stammen von STANDFuss. "Was. 
uns hier praktisch im allerhöchsten Grade interessiert, ist die bei einigen hierher gehörenden Formen durch vielfache Zucht bewiesene Tatsache, daß sie, mit der Grundart gekreuzt, keine Zwischenformen liefern, sondern daB die Nachkommenschaft wieder scharf geschieden - und zwar in beiden Geschlechtern - in die abweichende Form und in die Grundart zerfällt" (Standfuss, Berliner Entomol. Z. 1886, S. 238/39). Also gewisse Aberrationen - und dazu rechnen die melanistischen - ergaben bei Rückkreuzung mit der Stammform eine Spaltung: einerseits erschien die Stammform wieder, anderseits die Aberration. Leider konnte damals STANDFUSS die Resultate noch nicht zahlentheoretisch im Geiste MENDELs auffassen. Vielleicht war es anch besser so, denn späterhin hat diese Verquickung viel Unheil angerichtet: J. Gross hat, da sich die Zahlen dem Mendelschema nicht fügten, einen besonderen STANDFussschen Vererbungstypus aufgestellt. Daranf wird zuriickzukommen sein.

Nun zeigte sich aber bald - und STANDFuss selbst erhielt auch hierin als erster die Resultate --, daß der Versuch nicht immer so eintönig verläuft. Wenn die Experimente in gleicher Weise wie oben angesetzt wurden, traten in einigen Fällen Zwischenformen auf. Aus diesem vom obigen gänzlich verschiedenen Resultat schloß STANDFuss rückwärts auf die verschiedene genotypische Konstruktion - so sagen wir heute! - der verwendeten P-Eltern: im ersten Falle (s. oben) sei die abweichende Form entstanden durch "sprungweise Verschiebung" = "Aberration oder Varietät"; im zweiten aber sei sie „eine durch allmähliche Verschiebung entstandene Lokalrasse". Cond so gelangt er in seinem "Handbuch" (1896) am Ende dazu, zwei "durchaus verschiedene Fortpflanzungsresultate" bei der Kopula von Grundart und abweichender Form festzustellen:

1. Grundart $\times$ durch allmähliche Verschiebung entstandene Lokalrasse

eine Reihe von Zwischenformen;

2. Grundart $\times$ sprungweise Verschiebung $=$ Aberration oder Varietät

„in vielen Fällen keine Zwischenformen“, sondern Grundart und Varietät.

Wie weit dieses zweite Gesetz für Aberrationen Anwendung finden könne, stellt er der Zukunft anheim. Wenn wir einmal von der Terminologie STANDFuss' und von seinen deszendenztheoretischen Speku- 
lationen gänzlich absehen, so bleibt in seinen Theorien ein richtiger Kern übrig, der sich mit modernen Ansichten wohl verträgt. Er hat fast intuitiv das Wesentliche erfaßt. Nur die Konstanz der Zahlenverhältnisse ist ihm entgangen und mußte ihm entgehen, da ihm nur kleine Zahlen vorlagen. Späterhin sind die Experimente von STANDFuss und auch die von ihm zitierten anderer Autoren reichlich herangezogen worden zur Stütze für allerhand Theorien. Von manchem dieser Versuche läßt sich sagen, daß STANDFUss weder richtig gekannt, noch auch richtig verstanden worden ist. Das wäre allein schon ein genügender Grund für die nachfolgenden Ausführungen. Es sind aber auch seitdem eine Reihe weiterer Experimente veröffentlicht worden. Hier sei nur die an sich wertvolle Versuchsreihe von ScHRÖDER erwähnt. ScHRÖDERs Spekulationen bewegen sich aber lediglich in deszendenztheoretischer Richtung. Von der Höhe heutiger Kenntnis aus ließe sich leicht sagen, daß in seiner Nomenklatur eine große Verwirrung herrsche.

Vererbungstheoretisch kann es gar keinem Zweifel unterliegen, daß der Melanismus eine ganz einzigartige Erscheinung ist. Er kann sowohl bloße nicht erbliche Modifikation sein als auch erbliche Mutation. Noch öfter ist er wohl in seiner realen Ausprägung das Ergebnis einer komplizierten Kombination mendelnder Faktoren. Es dürfte zurzeit wenig andere Erscheinungen geben, die erblich so prinzipiell verschieden zu bewerten sind, vor allem keine, die so oft das Resultat einer Mutation ist. Daß die Untersuchung außerordentlich schwierig ist, vermag daran nichts zu ändern. Andererseits mag es diese große Bedeutung rechtfertigen, wenn in dem folgenden Versuch einer vererbungstheoretischen Analyse der Gesamterscheinung monographisch alle bisherigen Erfahrungen mit verwertet sind.

\section{Der Begriff des Melanismus.}

Die Schmetterlingszüchter unterscheiden zwischen Melanismus und Nigrismus. Letzterer soll entstehen durch Ausdehnung der schwarzen Zeichnung und sich von bloßer Potenzierung einzelner Zeichnungselemente bezw. Konfundierung derselben steigern bis zu absolutem Nigrismus. Dagegen sei Melanismus eine gleichmäßig über Körper und Flügel bis zu völliger Schwärzung sich erstreckende Einfarbigkeit, die von der Zeichnung völlig unabhängig ist. STANDFuss bezeichnet Melanismus als eine Neigung zur Verdüsterung der Färbung, die sich bis zu fast reinem Schwarz steigern kann, aber auch alle möglichen Zwischenstufen 
bis zur normalen Färbung hinab aufweist. Es gibt nun in der Tat einige Beispiele, die an eine Trennung der beiden Begriffe denken lassen. So bildet STICHEL in seiner Fig. 24 eine Lymantria monacha L. ab, die bei Bad Elm gefangen worden ist. Soviel der schauerliche Holzschnitt erkennen läßt, sind auf dem linken Flügel die schwarzen Zeichnungsbänder teilweise verbreitert, teilweise zusammengeflossen. Ganz anders der rechte Flügel. Darüber liegt es wie ein Hauch, unter dem die Zeichnung der typischen Form deutlich durchschimmert. Im linken Flügel soll es sich demnach um Nigrismus, im rechten um Melanismus handeln.

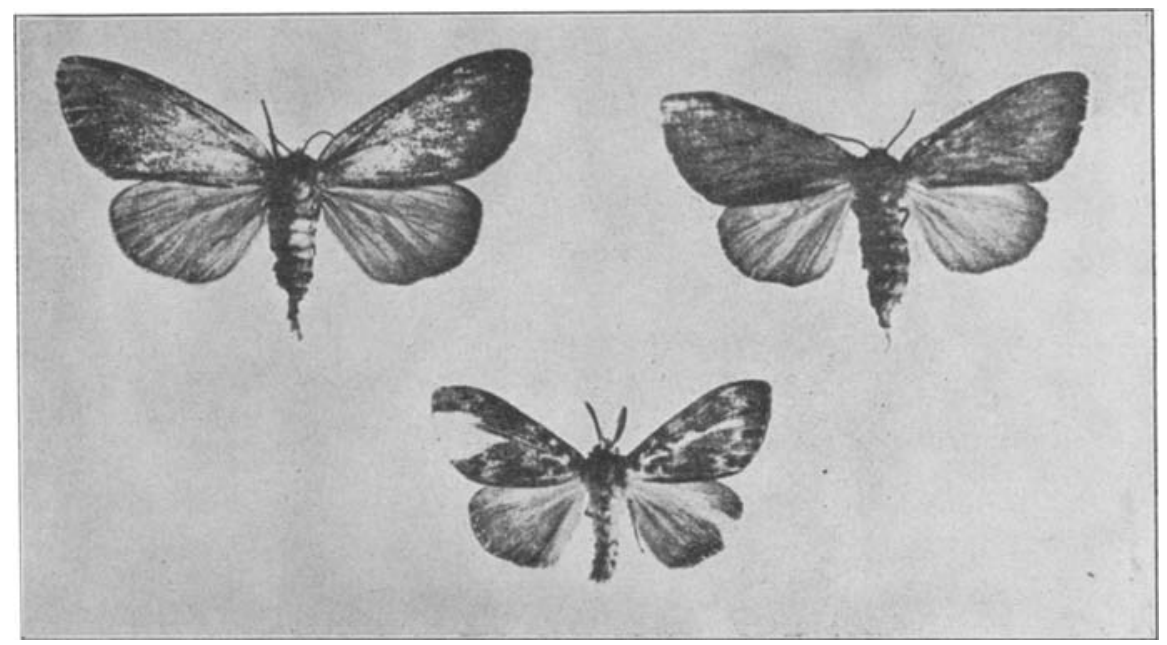

Fig. 1. Oben: 2 Iymantria monacha ab. eremita aus der Natur, das linke Exemplar etwas abgeflogen, sogenannte "typische" Stücke ohne Reste von Zeichnungselementen. Unten: Aberration und Stammform unsymmetrisch gemischt, wahrscheinlich ähnlich den 6 Stück von STaNdFUSS (1893).

Eine solche Unterscheidung, die sich nur an Hand einiger Paradebeispiele durchführen läßt, beruht auf einer ganz äußerlichen Auffassung der Sache, auf der Topographie. Das Pigmentierungsphänomen ist aber eine physiologische Erscheinung, die sich allenfalls noch histologisch studieren lieBe. Es haben sich denn auch eine Reihe von Autoren gegen eine Trennung der Begriffe ausgesprochen, so SCHULZE, SCHRÖDER, v. LINSTOW u. a., SPENGEL aber und teilweise StANDFuss halten daran fest. STicheL geht, obgleich er die prinzipielle Trennung verwirft, sogar so weit, für jede Stufe der Ausbildung besondere Termini aufzustellen, die natürlich schon vor der Aufstellung überflüssig waren. Begriffe, die sich nicht 
definieren lassen, sind immer überflüssig. In gewissen Grenzfällen ließe sich rein morphologisch ein Gegensatz konstruieren, prinzipiell ist er nicht berechtigt. Die Übergänge fließen ineinander ohne jede Grenze.

Diese Dinge müssen hier besprochen werden, weil sie mit den Vererbungsfragen verquickt worden sind und dadurch, weil selber unklar und verworren, notwendig Irrtum gestiftet haben. STANDFuss gehört zunächst zu der Reihe ScHulze, ScHRöDER, v. Linstow (siehe oben!). Auch er gibt zu, daß sich rein äußerlich Melanismus und Nigrismus nicht trennen lassen. Deswegen zieht er als Kriterium ein ganz neues Moment herein: das erbliche Verhalten. Aus der Färbung der Nachkommen will er Schlüsse ziehen auf die Ursache. Spalten diese rein in Stammart und Aberration, dann soll es sich um durch Sprungvariation entstandenen echten Melanismus handeln, erscheinen dagegen intermediäre Formen, dann um durch fluktuierende Tariabilität erzeugte Nigrismen. Es liegt klar, daß dann die beiden Begriffe ihren ursprünglichen Sinn vollständig verlieren und zu ganz neuen Kategorien gehören. Ist es aber schon an sich mißlich, morphologische Termini genetisch zu fundieren, so genügt es zur Charakterisierung dieses Verfahrens, auf die Konsequenzen hinzuweisen. Im übrigen ist hier der Begriff des Melanismus im weiteren Sinne gebraucht. Es sind darunter alle Potenzierungen der schwarzen Elemente über das normale Maß hinaus verstanden.

\section{Melanismus in Popnlationen und reinen Linien.}

Das interessanteste Objekt, das in dieser Hinsicht betrachtet werden kann, ist die Nonne. Im Jahre 1907 wurde Lymantria in Leipzig und Umgebung reichlich gefangen. Eine Durchsicht dieses Materials ergibt alle Übergänge von der normalen Form bis zu der völlig geschwärzten, auch die feinsten Nuancen sind manifestiert. Auf Tafel I ist eine solche Serie zusammengestellt, links Männchen, rechts Weibchen. Es erhebt sich die Frage nach der genotypischen Natur der in einer solchen Population gegebenen Ausbildungsgrade von weiß zu schwarz. Darüber kann nur das Experiment entscheiden. Cnd das ist bei bisexuellem Material nicht so einfach. So können z. B. zwei Individuen eines mittleren Ausbildungsgrades phänotypisch völlig gleich aussehen und doch genotypisch zwei verschiedenen Linien angehören. Mit solchen Tieren ist die erstrebte Isogenie schwer zu erreichen. Zweckmäßiger ist es, von den Plus- und Minusabweichern auszugehen. Darüber liegt ein einziges Experiment vor, das GoLDschmmT in seiner "Einführung" mit- 
teilt. Er wählte aus einer Population ein schwarzes Paar aus und erhielt als Nachkommen eine Variationsreihe von ganz schwarz wie die Eltern bis herunter zu einem Individuum, das deutlich schwarz und weiß gebändert ist und etwa einer Nuance zwischen dem 3, und 4. Weibchen (von oben) auf Tafel I entspricht. Stellt er Klassenvarianten auf und bezeichnet das eben beschriebene Tier als 1 , die ganz schwarzen mit 5 , dann verteilen sich die 39 Nachkommen wie folgt:

$$
\begin{array}{ll|l|r|r|r}
\text { Klasse der Färbung: } & 1 & 2 & \mathbf{3} & 4 & 5 \\
\text { Zahl der Individuen: } & 6 & 9 & \mathbf{1 3} & 7 & 4
\end{array}
$$

Damit hatte er, wie sich weiterhin zeigte, eine reine Linie isoliert. Weitere Selektion konnte den Plänotypus nicht mehr verschieben. Aus diesem leider einzigen Experiment in Verbindung mit den gleich zu besprechenden von SCHRÖDER ist immerhin eins sicher zu entnehmen: in einer Population der Nonne lassen sich mehrere, vielleicht viele reine Linien isolieren. Da aber transgressive Variabilität herrscht, kann natürlich die phänotypische Beschaffenheit nichts aussagen über die genotypische Zugehörigkeit. Also würde eine sehr große Zahl von Versuchen dazu gehören, um eine Nonnenpopulation zu analysieren.

Eine Bemerkung sei noch an GoLDschMmTs Versuch angeschlossen! Die 39 Nachkommen ordnen sich in eine symmetrische Variationsreihe. Mit dem Genotypus ist die Reaktionsnorm gegeben. Sie wird unter andern Verhältnissen anders ausschlagen. Was sich nicht verschiebt, ist lediglich der Mittelwert. Eine der Individuenzahl nach asymmetrische Reibe ist sehr leicht denkbar, im vorliegenden Fall noch aus einem besonderen Grunde. Denken wir, der Mittelwert einer reinen Linie läge bereits in Schwarz, dann werden die Plusabweicher nicht wahrzunehmen sein; denn unser Ange vermag schwärzer von schwarz nicht zu unterscheiden. Folglich müssen alle Individuen rechts rom Mittelwert zusammengeworfen werden nnd machen die Kurve asymmetrisch. $\mathrm{Ja}$, die Frage ist nicht müßig, ob es nicht eine reine Linie ganz innerhalb des Schwarz geben kann. Eine solche zu isolieren und dann mit einer weißen Linie zu kreuzen, wäre im Hinblick auf die späteren Darlegungei von außerordentlichem vererbungstheoretischen Interesse. Ihre Existenz kann kaum bezweifelt werden. Sehen wir doch z. B. bei Jennings, daß die eine reine Linie seiner Paramaecium-Kulturen mit ilhren Extremen den Mittelwert der Population nicht mehr erreicht!

Viel älter wie GoLDschurdTs Experiment mit Lymantria, das bier nur seines einfachen Charakters wegen zuerst angeführt wurde, sind 
Versuche von ScHRÖDER an Abraxas grossulariata L. Aus den zahlreichen Versuchen, die er (1903) mitteilt, sind vererbungstheoretisch nur die Reihen $\mathrm{A}$ und $\mathrm{C}$ wichtig.

Die Versuchsreihe A geht aus von einem ziemlich stark melanistischen Paar, das ScHröDER 1899 fand. Er rechnet es zu Klasse - IV. Hierbei sieht er die normale Form als Klasse 0 an und bezeichnet Aufhellungen als $+\mathrm{I},+\mathrm{II}$ usw., Verdunkelungen als $-\mathrm{I},-\mathrm{II}$ usw. Jenes Pärchen lieferte 1900 über 100 Puppen, die in zwei Portionen geteilt wurden. Die erste blieb unter normalen Verhältnissen ( $\mathrm{Ia}$ ) und gab 58 Falter. Die zweite wurde extremen Temperaturen ausgesetzt und brachte 39 schmetterlinge (Ib).

\begin{tabular}{|c|c|c|c|c|c|c|c|c|c|c|c|}
\hline 0 & $-I$ & $-I I$ & - III & $-I V$ & $-V$ & $-V I$ & - VII & -VIII & $-\mathrm{IX}$ & & \\
\hline 24,14 & 34,48 & 22,41 & 13,79 & 5,17 & & & & 513 & 956 & I a & 1900 \\
\hline 46,15 & & 5,13 & 5,13 & 7,69 & 10,26 & 2,56 & 0 & 5,13 & 2,56 & 10 & \\
\hline 14,52 & $\begin{array}{l}27,42 \\
24,14\end{array}$ & $\begin{array}{l}30,65 \\
31,03\end{array}$ & $\begin{array}{l}17,74 \\
20,69\end{array}$ & $\begin{array}{l}\mathbf{6 , 4 5} \\
4,5\end{array}$ & 1,61 & $\begin{array}{l}1,61 \\
23\end{array}$ & \multirow{3}{*}{2,74} & & & II a & 1901 \\
\hline 5,49 & 20,54 & 26,03 & 28,77 & 12,33 & 1,37 & 2,74 & & & & III a & \multirow{2}{*}{1902} \\
\hline 9,75 & 19,51 & 31,71 & 29,77 & 7,32 & 0 & 2,44 & & & & III b & \\
\hline
\end{tabular}

Wenn wir zunächst von dèr Schwierigkeit bisexuellen Materials, d. h. von möglicher oder besser wahrscheinlicher Heterozygotie ganz absehen, dann wäre die Verschiebung nach der Plusseite in Ib so aufzufassen, daß erst mit dem Temperaturexperiment die volle Reaktionsnorm aus dem Organismus herausgeholt worden ist. Zur Vollständigkeit wäre allerdings zu fordern, durch andere Bedingungen auch nach der Minusseite das Mögliche herauszuholen. Vielleicht darf dies jedoch in dem Falle unterlassen werden, da gerade bei Lepidopteren für die Aberrationen nach beiden Seiten keine spezifischen Einflüsse nötig sind. Das vermögen z. B. FIschers Temperaturexperimente mit Vanessa antiopa zu zeigen, wo Frost und Hitze, bezw. Kälte und Wärme, die gleichen Bewirkungen haben.

In graphischer Darstellung ergibt Ib eine zweigipflige Kurve, der eine Gipfelpunkt liegt in Klasse 0, der andere in Klasse - V. Offenbar müssen wir auf zwei verschiedene Linien schließen, deren jede zurgenüge durch eigenartige Modifikabilität charakterisiert ist. Die rechte reagiert in der normalen Weise auf die gegebenen Bedingungskonstellationen, sie folgt in ihrer Anordnung nahezu der Zufallsreihe. Nicht so die linke Linie, die eine auffällige Asymmetrie dartut. Ich streifte 
schon oben die hypothetische Möglichkeit asymmetrischer Kurven. Hier ist sie tatsächlich gegeben. Diese Linie reagiert ganz anders auf die äußeren Bedingungen wie die andere. Während letztere eine Potenzierung in melanistischer Richtung zeigt, bewegt sich erstere gerade entgegengesetzt, wie ein Vergleich nit der normalen Parallelkultur (Ia) erkennen läßt. Es ist anzunehmen, daß die linke Linie von Klasse 0 etwa bis zu Klasse - I oder auch - II reicht. Thre erbliche Reaktionsnorm ist derart, daß unter der Wirkung der Temperatur eine Verminderung der melanistischen Elemente eintritt, daher die Zunahme der Individuen in Klasse 0 gegeniiber der Normalkultur (Ia). So hat das Temperaturexperiment zweierlei klarzulegen vermocht: einmal, daß wir es hier überhaupt mit bloßer Modifikabilität zu tun haben, zam andern, daß hier ein Gemisch zweier Linien vorliegt. Vorsichtigerweise prüfen wir auch in dieser Hinsicht die späteren Generationen. Aus Ia hat SCHRÖDER ein Pärchen zur Nachzucht bestimmt, das Klasse - IV und damit wahrscheinlich der (in $\mathrm{Ib}$ ) rechten Linie angehört. Tatsächlich liegt in $\mathrm{F}_{2}$ und $\mathrm{F}_{3}$ der Gipfel konstant etwa in Klasse - II, eher etwas mehr nach - III, die geringen Schwankungen in der Verteilung kommen bei dem immerhin kleinen Material nicht in Frage. Da ScHRöDER auch weiterhin Klasse - IV zur Zucht benutzte, also Selektion in melanistischer Richtung ïbte, ist deren Erfolglosigkeit glänzend dargetan. Wir dürfen demnach behaupten, eine reine Linie isoliert zu haben. Dabei weise ich noch ganz besonders anf die verschiedene Lage des Kurvengipfels in Ia gegenüber den späteren Generationen hin, dort liegt er in - I, hier, wie schon gesagt, in - II mit Hinneigung zu - III. In Zucht Ia ist eben die eine Linie noch nicht eliminiert, die sich in $\mathrm{Ib}$ mit einem Gipfel in Klasse 0 bemerkbar macht.

SCHRÖDER glaubt dargetan zu haben, "daß ein (biologischer) Charakter in Verlaufe der Generationen nicht nur an Festigkeit gewinnt, sondern anch eine höhere Ausprägung erfahren kann." „Es ist aber nicht allein die Zunahme im prozentualen Auftreten der Aberrationen mit steigender Zahl der Generationen, es ist auch das Auftreten intensiverer Aberrationen gleicher Richtung ein [so] regelmäßiges" .... Mit anderen Worten: ScHr. glaubt an einen Erfolg der Selektion. Worauf in Wirklichkeit die Verschiebung des Kurvengipfels beruht, hat die obige Analyse ergeben. Von einem Erfolg der Selektion kann keine Rede sein. Sodann behauptet SCHR. die Erblichkeit der individuellen Variationen, auch der durch Temperaturexperimente erzielten. Er bezieht sich dabei gerade auf die hier mitgeteilten Versuche. Ich kann darin gar nichts 
Beweisendes finden. Übrigens hätte er eine ausgezeichnete Gelegenheit gehabt, sich von seiner irrtümlichen Ansicht zu befreien, wenn er nämlich in Versuch 1b Tiere der Klassen VIII und IX zur Fortpflanzung gebracht bätte. Wahrscheinlich würde dann die Kurve in Ia ebenso wie jetzt ausgesehen haben.

\begin{tabular}{|c|c|c|c|c|c|c|c|c|c|c|}
\hline$+\mathrm{II}$ & $+\mathbf{I}$ & 0 & $-\mathrm{I}$ & -II & $-\mathrm{III}$ & - IV & $-\mathrm{V}$ & $-\mathrm{VI}$ & $-\mathrm{VII}$ & \\
\hline & 18,03 & $\begin{array}{l}78,69 \\
57,14\end{array}$ & $\begin{array}{r}3,28 \\
23,81\end{array}$ & 0 & 4,76 & 4,76 & 4,76 & 0 & 4,76 & $\left.\begin{array}{l|l}\text { I a } & 1900 \\
\text { Ib }\end{array}\right\}$ \\
\hline 5,41 & 20,27 & 72,98 & 1,35 & & & & & & & II $>1901$ \\
\hline
\end{tabular}

Eine zweite Zuchtreihe geht von einem aufgehellten Pärchen aus, das ScHR. in Klasse + I stellt. Die Nachkommen des Paares wuchsen ebenfalls unter getrennten Bedingungen anf und gaben bezüglich 61 (Ia) und 21 (Ib) Falter. In Ib tritt wieder eine stark asymmetrische Kurve auf, die aber im Gegensatz zu vorhin eingipflig ist. Ein Pärchen der Klasse 0 von Ia brachte 190174 Tiere hervor (II). Leider schlugen die Versuche fehl, $F_{3}$ zu erhalten. Doch trage ich keine Bedenken, zu sagen: in Zuchtreihe $\mathrm{C}$ liegt ebenfalls eine reine Linie vor, da die Ausschläge in Ib bis zu Klasse VII, wie schon die geringen Individuenzahlen beweisen, nur persönliche Fluktuationen sind. Immerhin würde unser Urteil darüber noch sicherer sein, wenn etwa in jeder Generation in gleicher Weise wie in I mit dem physiologischen Experiment gearbeitet worden wäre. Hier erscheint dies direkt als Hilfsmittel der erblichen Analyse, wenigstens nach der phänotypischen Seite hin. Das sicherste Mittel bleibt natürlich unter so schwierigen Verhältnissen die fortgesetzte Auswahl extremer Plus- oder Minusabweicher für die Zucht.

\section{Versuche über Kreuzung von reinen Linien.}

In freilich ganz anderer Absicht hat ScHRöDER die eben besprochenen reinen Linien gekreuzt. Dieses Experiment ist vererbungstheoretisch von sehr großem Interesse, weil zoologisches Material über diesen Punkt anderweit kaum vorliegt, während die Botanik gerade darin wahre Triumphe sorgfältigster Untersuchungen gefeiert hat.

Aus Ib der Zuchtreihe A nahm ScHR. ein ơ der Klasse - IX, also eine sehr stark melanistische Form, und kreuzte sie mit einem o der Reihe C aus Ib, das der Klasse - VII zugehört, mithin auch sehr dunkel ist und dem Geschlechtspartner recht nahe steht. Ehe wir das Resultat 
betrachten, wollen wir sogleiclı das Elternpaar einer analogen Hybridisation kennen lernen. Beide Tiere stammen aus den gleichen Linien. Nur gehören sie den Klassen - $\mathrm{I}^{r}$ und - VI zu.

\begin{tabular}{|c|c|c|c|c|c|c|c|c|c|}
\hline$+I$ & 0 & $-I$ & - II & - III & $-I V$ & $-\mathrm{V}$ & $-V I$ & $-V I I$ & \\
\hline 2,63 & $\begin{array}{l}\mathbf{3 5}, \mathbf{5 3} \\
21,18\end{array}$ & $\begin{array}{l}27,633 \\
\mathbf{3 0 , 5 9}\end{array}$ & $\begin{array}{c}9,21 \\
24,7\end{array}$ & $\begin{array}{r}15,79 \\
7,06\end{array}$ & $\begin{array}{r}2,63 \\
12,94\end{array}$ & $\begin{array}{l}5,26 \\
3,53\end{array}$ & 0 & 1,32 & $\begin{array}{ll}\mathrm{K}_{1} & 1901 \\
\mathrm{~K}_{2} & 1901\end{array}$ \\
\hline
\end{tabular}

Die graphische Darstellung ergibt eine zweigipflige Kurve in beiden Fällen. Sie ist hier der phänotypische Ausdruck für die beiden in der Hybridengeneration enthaltenen Linien. Allerdings bleibt ein bitterer Rest von Ungeklärtem, warum nämlich in $K_{1}$ und $K_{2}$ die rechten Gipfel so sehr niedrig liegen. Möglich, daß es einfach auf ungleiche Repräsentation der beiden Linien zurückgeht. Da aber die Betrachtung der Kurven nichts aussagt über die genotypische Beschaffenheit, da ferner zweigipflige Kurven der Ausdruck für verschiedene Möglichkeiten sind, so ist es sehr zu bedauern, daß die extremen Abweicher nicht weiter gezüchtet worden sind.

Noch ein anderer Punkt sei kurz erörtert. Trotzdem das Elternpaar von $\mathrm{K}_{1}$ den Klassen - VII bezw. - IX angehörte, ist von einer Selektion nichts zu bemerken. Im Gegenteil sind die Klassen - VIII und - IX gar nicht vertreten. Im Verein mit dem Ausfall von $\mathrm{K}_{2}$ ist das ein schöner Beweis, wie nicht die Modifikationen als solche erblich sind, sondern lediglich die Modifikabilität oder die Reaktionsnorm. SCHRÖDER bezeichnet jenes Elternparar ils „stark divergente, im Gesamthabitus mutierte Formen". Sie seien unter dem Einfluß extremer Temperaturen in weitestgehender Mutation yon normalen Formen auf kaum noch zu überschreitende geschnellt. Infolgedessen erwartet er nach DE VRIESscher Hypothese erbliche Koustanz. Diese trat aber nicht ein. Daraus mußte ScHRöDEn rückwärts auf eine andere erbliche Natur des Organismus schlieBen. Das tat er nicht, wundert sich vielmehr über die Inkonstanz der Mutation und findet so Gelegenheit, gegen DE VRIEs zu polemisieren. Wir wissen heute, daß3 die Entfernung einer Aberration von der Stammform - und sei sie gleich recht groß - noch gar nichts anssagt ïber die genotypische Struktur und damit das erbliche Verhalten. Oftmals liegen Mutanten der Ausgangsform so nahe, daß sich die Modifikationskurven beider überschneiden. SCHRÖDER übersieht hier vollständig eine Qualität, die bereits JE T'RIES untrennbar mit dem Begriffe der Mutation verbunden liatte: den erblichen Charakter. 
Er erfaßt lediglich die phänotypische Seite der Sache, nicht die genotypische.

Wir wenden uns nunmehr zu einer andern Versuchsreihe ScHRöDERs, die er einige Jahre später (1908) veröffentlicht hat. Sie betrifft Lymantria monacha. Auch sie mub unter der Perspektive der Kreuzung reiner Linien betrachtet werden, wenn sie nicht völlig unverständlich bleiben soll. Freilich bringt sie gegenüber den Abraxas-Experimenten keinen Fortschritt. Sie arbeitet mit unreinem, nicht definiertem Material.

Zunächst kreuzte ScHRÖDER wie folgt:

\begin{tabular}{|c|c|c|c|}
\hline \multirow[b]{2}{*}{ Resultat: } & \multicolumn{3}{|c|}{ Lymantria monacha of $\times$ L. ab. eremita } \\
\hline & $\underbrace{180^{7} 119}$ & $90^{\pi} 15$ 오 & $250^{\pi} 469$ \\
\hline
\end{tabular}

Erinnern wir uns, wie STANDFuss das Ergebnis beurteilt hätte! Ohne Zweifel hätte er das eremita-ơ als „eine durch allmähliche Verschiebung entstandene Lokalrasse" angesprochen; denn nur dann kann die Rückkreuzung mit der Grundart Übergänge ergeben. Nicht so ScHRÖDER. Er fing gleichzeitig 43 Tiere und zwar monacha, von denen er eins in Fig. $2 b$ wiedergibt: es ist schwach melanistisch; außerdem fing er ein typisch melanistisches $\sigma^{7}$, das der obigen Kreuzung. Und dieses Individuum mit seinem verhüllenden Schwarz von vollkommener Diskretion erklärt er für eine typische Mutante. „Hier bitte ich nur darauf hinweisen zu dürfen, daß eine zweifellos typisch melanistische und aller Denkbarkeit nach mutierte Form bei Kreuzung mit der normalen in der Nachkommenschaft nicht rein gespalten ist, sondern daß auch gewöhnliche Zwischenformen neben mosaikartig gebildeten aufgetreten sind. Zwar neige ich auch der Ansicht zu, daß manche Melanismen rein spalten, es dürfte das aber nicht mit dem Begriffe des Melanismus zu verbinden sein." Wo in aller Welt liegt der Beweis dafür, daß es sich um eine aller Denkbarkeit nach mutierte Form handelt, wenn nicht darin, daß zu wenig gedacht wurde? Es fehlt doch jeder, anch der geringste Anhalt. Der genotypische Charakter kann einer im Freien gefangenen Form niemals angesehen werden. Darüber entscheidet einzig und allein das Vererbungsexperiment, und das zeigt das Gegenteil von dem, was ScHRÖDER behauptet. Er hatte nur nötig, auf STANDFUSS zurückzugreifen, dann boten sich ihm für die Interpretation gar keine Schwierigkeiten. Wenn wir nun nach den Ursachen für ScHRöDERs Irrung suchen, dann sind die leicht zu finden: sie liegen in der schon oben besprochenen unseligen Verquickung von morphologischen und genetischen Begriffen. 
Er hat assoziiert "typischer Melanismus" und Mutation. Eine typisch melanotische Form braucht aber keine Mutante zu sein. Es kann sich ebenso gut um das extreme Endglied einer Variationsreihe handeln.

Überblicken wir nunmehr ScHRÖDERs Experimente in ihrer Gesamtheit!

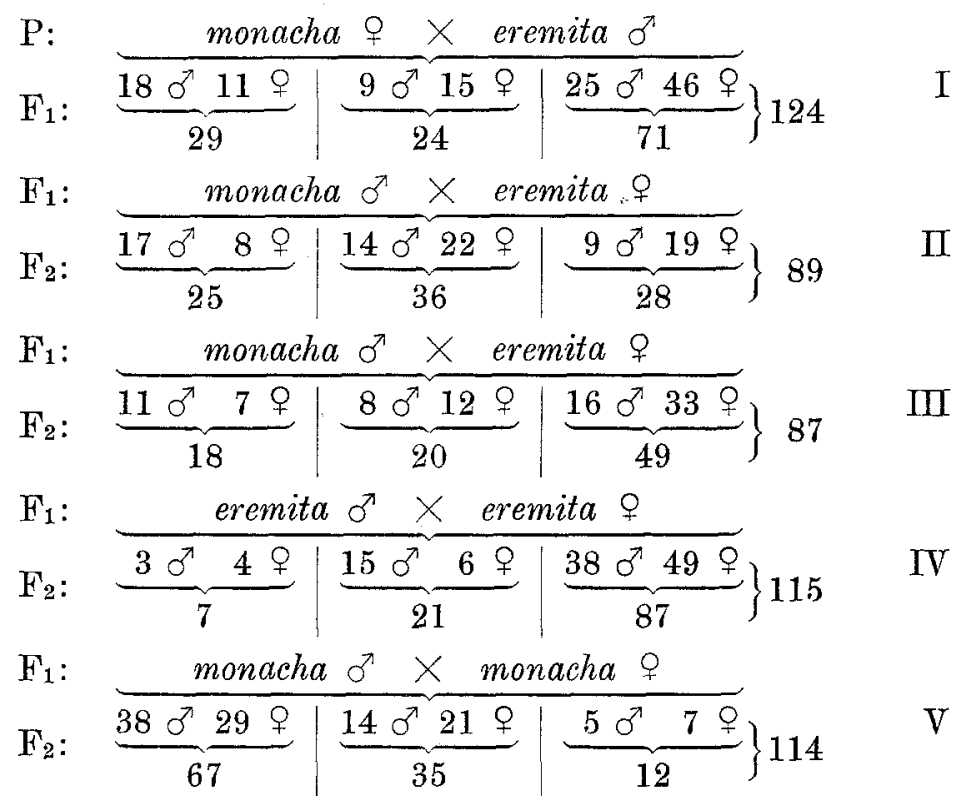

Zunächst ist eines zu erinnern. Nur die Tiere des I. Versuchs stammen aus dem Freien. Die Ausgangstiere der andern vier Versuche sind in I erhaltene $F_{1}$. Das P-eremita- $\sigma^{\top}$ ist äußerlich ein typisch melanistisches Exemplar. Es wird in Fig. 2 a von SCHRÖDER reproduziert. Aus den $\mathrm{F}_{1}$-Zahlen ließe sich ohne jeden Zwang ein Verhältnis von $1: 1: 3$ herauslesen. Die große Frage ist nun die, ob diesen Zahlen Wert beigelegt werden darf. Hier kann nur die kritische Betrachtung der $\mathrm{P}$ weiterbringen. Und da ist klar, daß Frei-Material aller Wahrscheinlichkeit nach nicht homozygot ist, wie ja oben die Betrachtung des AbraxasFalles ergab. Das $\mathrm{P} q$ kann selbst sehr gut einer Kreuzung zwischen monacha und eremita entstammen. Die gleiche Unsicherheit besteht für das $\mathrm{Po}^{7}$. Etwas besser sind wir in den folgenden Versuchen daran, da die Ausgangstiere als Plus- oder Minusabweicher der $F_{1}$-Reihe doch einigermaßen definiert sind. Bei immerwährender Auswahl der extremen Abweicher muß ja die Reinheit zunehmen. 
Das eremita-o des Versuches II wird in Fig. 2c abgebildet. Es ist keine typisch melanistische Aberration, sondern gehört eher zu den dunkelsten Übergangsstücken. In $\mathrm{F}_{2}$ verhalten sich die Gruppen etwa wie $1: 1: 1$. In Versuch III wurde ein "eremita-Typus ${ }_{+}$" benutzt, und es ist immerhin von Belang, daß sich sofort in $\mathrm{F}_{2}$ das Verhältnis $\mathrm{zu}$ $1: 1: 2^{1 / 2}$ (bezw. 3) verschiebt. Irgendwelche weiteren Schlüsse zu ziehen, wäre von Übel. Besonders interessant ist Versuch IV und V ausgefallen. Ein eremita-Pärchen weist unter seiner Nachkommenschaft weiße Stücke auf, typische monacha. In Hinsicht auf das oben von GoLDschmmT mitgeteilte Experiment sind die beiden Eltern oder wenigstens eins als heterozygot anzusprechen. Ein eremita-Individuum, das einer reinen Linie angehört, dürfte kaum bis zu völligem Weiß „persönlich fluktuieren". Das Verhältnis in $\mathrm{F}_{2}$ ist $1: 3: 12$. Es ist recht interessant und erinnert sofort an einen ganz bestimniten Fall, in dem gleichsinnige Faktoren eine Rolle spielen. Wenn nämlich zwei Faktoren vorhanden sind, wovon der eine den andern verdeckt, tritt die gleiche gegenseitige Beziehung hervor. Vielleicht handelt es sich hier nicht bloß um eine äußere Analogie. Dafür spricht auch das Ergebnis von 6:3:1 in V. Nur daß wir glücklicherweise das Vermögen des monacha-Typus viel genauer kennen. Unter den Nachkommen reiner monacha treten keine eremita auf. Also ist in $\mathrm{V}$ wenigstens ein Tier heterozygot gewesen.

So muß gesagt werden, daß sich mit diesen Experimenten SCHRöDERs wenig anfangen läßt, da sie mit unreinem, wenig definiertem Material arbeiten ${ }^{1}$ ). Aber der Weg für zukünftige Untersuchungen auf diesem Gebiete läßt sich daraus klar entnehmen. Die Nonne zeigt gegenwärtig stark fluktuierende Variabilität in der Richtung von Weiß auf

1) Schrö̀le (1909) hat zwei Pärchen der "typisehen ab." von Amphidasys gekreuzt, also doubledayaria, und die Nachkommen unter verschiedenen Bedingungen aufgezogen: unter günstigen und schlechten. Die Einwirkung auf die Tiere versuchte er exakt zu fassen durch Messung der Flügelspannung. Unter günstigen Verbältnissen liegt der Hittelwert für Stammform und $a b$. bei $24 \mathrm{~mm}$, unter schlechten für erstere bei reichlich $22 \mathrm{~mm}$, für letztere bei $26 \mathrm{~mm}$. Wenn die Versuche die „konstitutionelle Prävalenz der Melanismen" beweisen sollen, dann muf der Verfasser seine Angaben ganz anders detaillieren. Ich will zur Kritik nur auf den Begriff „Reaktionsnorm" und auf WoLTERECKs Phänotypenkurven hinweisen. Unerläßliche Voraussetzung für solche Versuche ist genau analysiertes Material. - Im übrigen ist es das einzige mir aus der Literatur bekannt gewordene Experiment, wo eine Kreuzung doubledayaria $\times$ doubledayaria Zwischenformen gegeben hat. Dann handelt es sich also, um das noch einmal zu wiederholen, nicht um die typische Aberration, wie ScI. will. Es wird zukünftig anf melanistische individuelle Variationen anch bei dieser Form zu achten sein. 
Schwarz. Die erste Aufgabe muß sein, möglichst reine Linien zu ziehen. Dann erst hat die Batardierung Sinn und verspricht Resultate, die sich theoretisch verwerten lassen.

Nur der Vollständigkeit halber sei hier noch das älteste Experiment dieser Art angeführt. STANDFuss fand 1883 bei Liegnitz in Schlesien ein eremita- $\sigma^{\top}$ in Paarung mit einem monacha-o. Dieses $\sigma$ gehörte aber nicht der typischen Aberration an, sondern besaß noch weißliche Zeichnungen an der Flügelbasis und einen rötlichen, nicht durchweg geschwärzten Leib. In $F_{1}$ traten auf teilweise normale monacha o und $q$, alle Übergänge und einige wenige eremita $\sigma^{7}$ und $\odot$. Genaue Zahlen kann StandFuss nicht geben.

Rückschanend auf diesen Abschnitt könnten die geringen positiven Resultate verwondern. Immerhin hat sich einiges Grundlegende für die vererbungstheoretische Auffassung des Melanismus herausgestellt. Die Variationsreihe von Weiß zu Schwarz, wie sie uns in einer Nonnenpopulation entgegentritt, ist nicht das Ergebnis einer einheitlichen Ursache. Letztlin führt die Analyse einer solchen Population auf reine Linien mit bestimmter Modifikationskurve, und es könnte sehr wohl daran gedacht werden, die phänotypische Mannigfaltigkeit durch diese Einheiten aufzulösen. Aber sie sind keine gegenseitig abgeschlossenen Biotypen. Vielmehr kreuzen sie sich bunt durcheinander und der Lymantria-Fall lehrt, wie dadurch eine reiche Variabilität zustande kommt. Umgekehrt ist es ungemein schwer, daraus isogenes Material zu isolieren. Im einzelnen mag die hybride Natur eines Organismus oftmals noch komplizierter liegen wie oben bei Abraxas (Versuchsreihe A). Aber der SchlnB liegt nahe, daßB bereits wenige Linien ausreichen würden, um jene Mannigfaltigkeit zu erzengen. Welchen internen Gesetzmäßigkeiten die Kreuzung ehemals reiner Linien unterliegt, ist hei diesen Ohjekten sehr schwer auszumachen. Ganz im allgemeinen ist jedoch (s. Lymantria!) der alternative Charakter gar nicht zu verkennen.

\section{Melanismns als Mutation und sein erbliches Verhalten.}

Am 6. Juni 1910 wurde in Stötteritz bei Leipzig ein recht ungleiches Pärchen in Kopulation gefunden: Amphidasys betularius L. nnd seine $a b$. doubledayaria Mill., hierbei eine Bemerkung. Es scheint überhaupt nicht selten vorzukommen, da $\beta$ sich Aberrationen mit der Stammform in Freien paaren. Das festzustellen ist in gewisser Hinsicht wesentlich. StandFuss berichtet einen Fall von der Nonne (1883) und 
macht es wahrscheinlich, daß die seltenen Abformen von Aglia tau, nämlich ferenigra und melaina, in Ermangelung ihres Geschlechtspartners immer mit der Stammform kopulieren. Ähnlich wie für den Nagelfleck liegt die Sache für den Birkenspanner. Dieser Punkt erhöht das Interesse am Kreuzungsresultat außerordentlich. Das o der erwähnten Paarung legte sehr reichlich Eier ab. Die Zahl wurde auf 600 geschätzt. Davon wurde die Hälfte zur Nachzucht bestimmt. Nach Überwinterung der Puppen schlüpften im Jahre 1911:

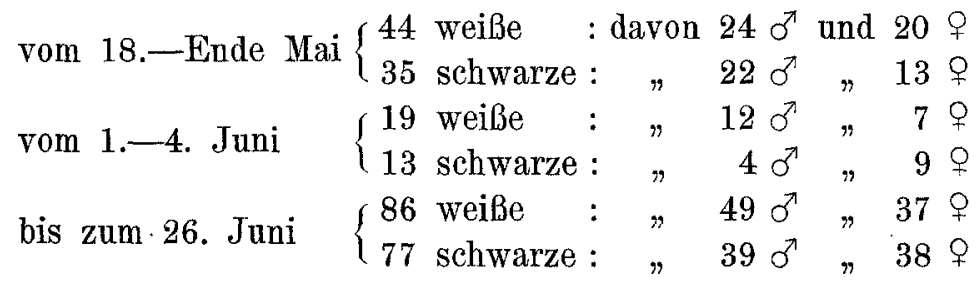

Die beiden letzten Tiere, 2 weiße $\sigma^{7}$, schlüpften am 5. und 6 . Juli. Das sind im ganzen 274 Schmetterlinge. Sie verteilen sich dem Geschlecht nach wie folgt: $150 \sigma^{\pi} \sigma^{7}$ und 124 $\%$, dem Aussehen nach: 149 weile und 125 schwarze. Also:

$$
\begin{aligned}
& \text { betularius } \sigma^{7} \times \text { doubledayaria } 9 \quad 1910
\end{aligned}
$$

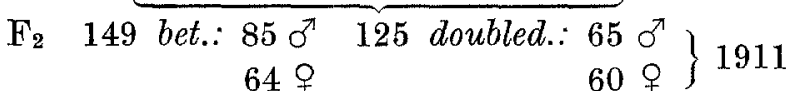

Auf Tafel II ist oben das elterliche Paar wiedergegeben. Das $q$ ist ein typisch melanistisches Exemplar. Früher ist nur die weiße Stammform bekannt gewesen. Die schwarze Aberration trat zuerst in England auf, dann in Westfalen und der Rheinprovinz, 1884 ist sie von Hannover und Gotha gemeldet, später von Dresden und 1892 von Gnadenfrei in Schlesien. Es ist nicht bekannt geworden, ob zwischen Stammform und Aberration irgendwelche Übergänge auftreten, wie das oben von Nonnenpopulationen geschildert wurde. Vielmehr erschien sofort die völlig geschwärzte Form, die zunächst recht selten war. Im Laufe der Jahre soll aber ihre Häufigkeit besonders in Großbritannien recht zugenommen haben. Jedenfalls $z$. B. besitzt sie aus Leipzigs Umgebung jeder Ortssammler. Ob und inwieweit die Stammform variiert, ließ sich hier nicht feststellen. Es ist aber durchaus erlaubt anzunehmen, daß sie fluktuierende Variabilität aufweisen wird. Freilich sind dafür die anf Tafel II wiedergegebenen $\mathrm{F}_{2}$-Stücke kein bündiger Beweis, denn oftmals tritt Variabilität erst infolge der Bastardierung hervor. Die 
6 Exemplare zeigen eine solch fortschreitende Potenzierung und Konfundierung der schwarzen Zeichnungselemente. Doch sind so dunkle Tiere wie reproduziert unter den 149 betularius der $\mathrm{F}_{2}$-Generation ganz selten. Sie können unter keinen Umständen als Übergänge zur melanistischen Form angesprochen werden: dazu sind sie erstlich zu wenig dunkel und zweitens zu wenig zahlreich. Die auf Tafel $D$ in Klassenvarianten aufgestellte Modifikationsreihe liegt durchaus innerhalb von betularius.

Und damit kommen wir zu dem Punkte, der das Resultat dieses Versuches so überaus interessant macht: zu der glatten Spaltung in Grundform und Aberration in $\mathrm{F}_{2}$. Die Erscheinung ist nicht neu. Schon STANDFuss und STEINERT haben sie gekannt. Aber sie haben vererbungstheoretisch nicht viel damit anzufangen gewußt. Für STANDFUSS war die Spaltung ein Fall sui generis, so auch noch für GRoss. Letzterer spricht deswegen von einem STANDFussschen Vererbungstypus. In $F_{1}$ (nach meiner Bezeichnung $F_{2}$, s. später!) sollen die Bastarde in beide elterliche Formen regelmäßig, aber in sehr wechselnden Zahlenverhältnissen „zurückschlagen“. Schon dieses letzte mystische Wort, das für eine exakte Forschung nicht vorhanden sein darf, kennzeichnet Gross' Ansicht zur genüge. Außerdem treffen beide tatsächlichen Angaben nicht zu, woraus aber GRoss kein Vorwurf zu konstruieren ist.

Vorerst, wie steht es mit der "irregulären Spaltung"? Da läßt sich in unserm Falle eine einfache Relation von $1: 1$ in den Zahlen 149 weiß : 125 schwarz gar nicht verkennen. Das Verhältnis wird noch deutlicher, wenn wir die andern bekannten Experimente heranziehen.

$$
\begin{aligned}
& 149 \text { betularius } 125 \text { doubledayaria. } \\
& \begin{array}{cc|ccc}
75 & n & 90 & & \\
8 & n & 7 & & \text { (STELNERT) } \\
\hline 232 \text { betularius } & 222 \text { doubledayaria }
\end{array}
\end{aligned}
$$

Das ist eine Beziehung wie 1,04:1, besser kann sich ein Verhältnis kaum aussprechen. Und dazu sind es recht lebensvolle Zahlen. Sie lassen sofort an das Rückkreuzungsschema denken, wie es uns sich eingeprägt hat von CORRENs' bekannter Darstellung zwischen einer rot und weiß blühenden Sippe her. Gekannt haben wir es natürlich schon vorher, seiner so sehr wichtigen Beziehungen wegen. Doubledayaria hat hier den Charakter eines Bastardes. Es ist heterozygot und bildet zweierlei Keimzellen: solche für weiß und solche für schwarz. Kommen 
die ersteren mit den Gameten der Stammform zusammen, so gibt es natürlich homozygote betularius. Wenn aber eine Gamete für schwarz mit einer für weiß sich vereint, entsteht die Aberration. Demnach ist schwarz dominant über weiß. Also:

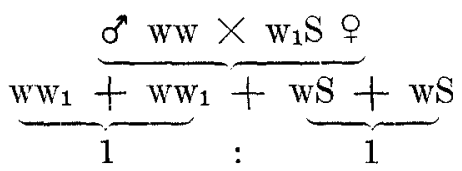

Diese reine Spaltung ohne das Auftreten von Übergängen zeigt, daß wir vererbungstheoretisch betrachtet einen neuen Fall vor uns haben. Hier hat die Aberration nicht mehr bloß den Wert einer individuellen Variation. Sie ist vielmehr eine Mutante, welcher Name nach vor- und rückwärts zugleich aussagt.

\section{Alternative Vererbung melanistischer Mutationen.}

ARNOLD LANG (Die Erblichkeitsverbältnisse der Ohrenlängen . .) hat einmal als charakteristisch für die alternative Vererbung die Uniformität der $\mathrm{F}_{1}$-Generation bezeichnet. Das ist richtig, trifft aber nur die phänotypische Seite der Sache. Letzten Endes ist alternative Vererbung bedingt durch das selbständige erbliche Verhalten der Faltoren, durch das, was wir Spaltung nennen. Diese aber kommt erst in $F_{2}$ zum äußeren Ausdruck. Wohl aber ist die Einheitlichkeit der $\mathrm{F}_{1}$ außerordentlich wichtig zur Orientierung in einer Experimentalserie und dies ganz besonders im Hinblick auf die verschiedenen Zahlenverhältnisse in $\mathbf{F}_{2}$. So milissen wir die Amphidasys-Generation, die die Spaltung in 1:1 aufweist, bereits als $F_{2}$ ansprechen. Die $F_{1}$ wird dargestellt durch die Mutante selbt. Jede doubledayaria ist eine "fliegende Heterozygotengeneration". Unter diesen Gesichtspunkten ist in dieser Arbeit die Bezeichnung der Generationen vorgenommen worden.

Demnach gehört der Amphidasys-Fall der typisch alternativen Vererbung an. Das beweisen auch verschiedene $\mathrm{F}_{3}$-Generationen, Mit $\mathrm{F}_{2^{-}}$ Tieren wurden die 3 möglichen Kombinationen vollzogen ohne Rücksicht auf die Reziprozität.

a) $\quad$ weißes Tier $=w_{w_{1}} q \times$ schwarzes Tier $=w_{S} \sigma \quad \mathrm{F}_{2}$

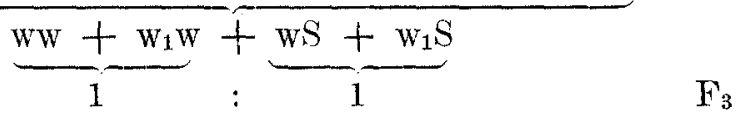


Erhalten- wurden 13 schwarze $(8 \circ 507$ ) $\left(\begin{array}{llll}10 & 9 & 3 & 7\end{array}\right)$, also genau das erwartete Resultat.

b) $\quad$ weißes Tier $=w_{w_{1}} \subseteq \times$ weißes Tier $=w_{w_{1}} \sigma^{\lambda} \quad \mathrm{F}_{2}$ $\mathrm{ww}+\mathrm{w}_{1} \mathrm{w}+\mathrm{ww}_{1}+\mathrm{w}_{1} \mathrm{w}_{1} \quad \mathrm{~F}_{3}$

Es sind in 1912 nur weiße Tiere erschienen, die Geschlechter zu gleichen Anteilen.

c) schwarzes Tier $=\mathrm{wS} \stackrel{9}{\circ} \times$ schwarzes Tier $=\mathrm{wS} \sigma^{7} \mathrm{~F}_{2}$ $\frac{w w}{1}: \underbrace{s w+w S+S s}_{3}$

Auch hier entspricht der Ausfall den theoretischen Erwartungen bez. Folgerungen: erhalten 1 weißes $\sigma^{3}$ und 4 scliwarze Tiere $\left(\begin{array}{llll}3 & \sigma^{7} & 1 & \%\end{array}\right)$. Auffallen könnten die geringen Zahlen dieser Versuche. Aber das ist verständlich aus der Fortpflanzungshiologie dieser Spanner. Sie überwintern als Puppen, und diese sind bekanntlich schwerer durch den Winter zu bringen wie Eier. Übrigens ïberwintern sowohl monacha wie dispar als Eier, daher die zum Teil hohen Zahlen in solchen Versuchen. Trotzdem genügen die Amphidasys-Tersuche jeder billigen Anforderung, da sich die Resultate in schöner C̈bereinstimmung in positiver Richtung bewegen.

Nenerdings hat HASEBROET ein interessantes Experiment C. ZIMLERMarns mitgeteilt. Im Jahre 1904 ist plötzlich bei Hamburg eine melanotische Mutation des Nachtfalters Cymatophora or F. anfgetreten, die den Namen $a b$. albingensis führt. Ihre Entstehung wird auf die lokale Einwirkung von Ruß und Ranch zurückgeführt. Davon ist hier abzusehen. Viel wichtiger ist, daß keinerlei Zwischenformen beobachtet wurden, vielmehr einzig die tiefschwarze Form. 1904 war sie sehr selten, 1911/12 wurden ans Raupen gewisser Fundstellen bis 90 und $95 \%$ erzogen. Znumermans hat gekreuzt wie folgt:

albingensis $\times$ albingensis.

Er hat erhalten 20 Exemplare albingensis und 6 der Stammform, das ist ansgesprochen die Relation von $3: 1$, demnach:

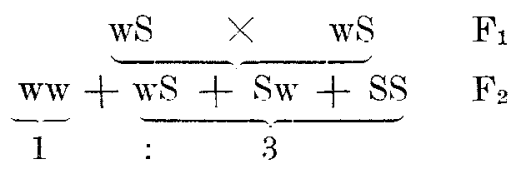


Also auch die jetzt in Entstehung begriffene Aberration albingensis ist heterozygot und bringt in $\mathrm{F}_{2}$ eine Spaltung, wie sie von echtesten Mendelfällen bekannt ist. Daß bei der gewaltigen Zunahme der $a b$. in gewissen Sektoren der Hamburger Umgebung bald homozygote Individuen auftreten werden, liegt auf der Hand. Es ist nicht überflüssig, die damit gegebenen Kombinationsmöglichkeiten zu überblicken.

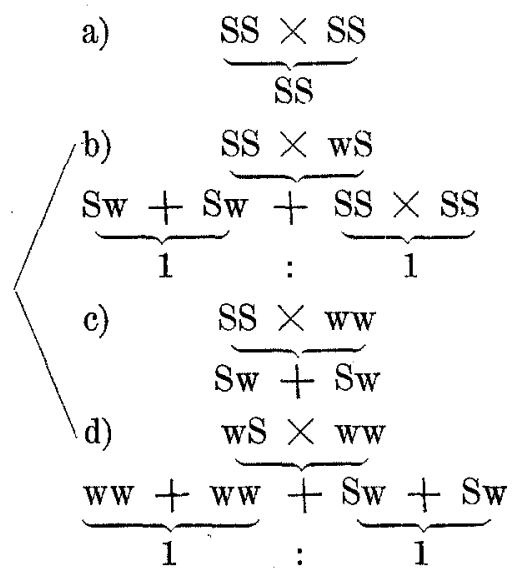

Wenn in Hamburg, wie es scheint, die Experimente systematisch durchgeführt werden, dann dürfen wir in einigen Jahren vollständige Klärung dieser Verhältnisse erwarten. Allerdings möchte dabei auf ganz andere Dinge mitgeachtet werden, denn schließlich liegt die Sache furchtbar durchsichtig. Es ist ein starrer Ablauf alternativer Vererbung ohne jede Besonderheit.

Am Ende sei noch kurz die bisherige Literatur auf solche Fälle durchgesehen. Da stoßen wir zunächst auf ein Experiment von STANDFUsS aus dem Jahre 1893. Er züchtete ein normales Paar von Lymantria monacha und erhielt unter zahlreicher Nachkommenschaft ein einziges vollkommen geschwärztes $q$, also ab. eremita $O$. Es wurde von einem normalen monach $\alpha-\sigma^{\circ}$ begattet und brachte Junge wie folgt: $2 \sigma^{\pi} \sigma^{\pi}, 20$ 우 monacha; $5 \sigma^{\pi} \sigma^{\pi}, 1$ 우 typische Form und $a b$. unsymmetrisch gemischt (s. Fig.); und $18 \sigma^{\pi} \sigma^{\pi}, 5$ 우 ab. eremita. Dies ist das einzige Experiment, in dem von der starren Regel eine kleine Abweichung in Gestalt der 6 unsymmetrisch gemischten Tiere auftritt. STANDFUSS schließt etwaige gynandromorphe Natur aus und hält sie für fortpflanzungsfähig. Hätte er nur eines dieser Tiere wieder mit der Stammform rückgekreuzt, dann würden wir ihre Natur ganz genau kennen. 
Ich vermute in ihnen Mosaikbastarde. Da sie in so geringer Zahl erscheinen, wäre weiterhin an eine geringe Potenzschwankung der Faktoren zu denken. Sie sind einander nicht unbedingt hierarchisch überlegen.

STANDFuss selbst gibt nichts anf die Zahlen dieses Versuches, da er mißglückte. Anders Gross (1906, S. 508): „Die Paarung gelang sehr gut ..." Die 51 Stück nennt er „eine immerhin beträchtliche zahlreiche Brut.“ Immerhin eine interessante Erscheinung, wie mangels anderer Belege vom Autor selbst gering eingeschätzte Versuche zu Wert und Bedeutung kommen. Es ist auch nicht gut angängig, wenn DE MEIJERE (1913) die kleinen Zahlen für geschlechtsbegrenzte Vererbung heranzieht, wiewohl das naheliegt. Hat doch GoLDSCHMD'T bei Kreuzung von monacha mit eremita reine Spaltung mit merkwürdiger Geschlechtsverteilung erzielt: alle weißen sind Weibchen, alle schwarzen hingegen Männchen. DE MEIJERE zitiert auch Versuche von BACOT und SMALLWOOD für geschlechtsbegrenzte Vererbung.

$$
\begin{aligned}
& \underbrace{\text { Amph. betularius }{ }^{7} \times \text { doubledagaria } \text { 우 }}_{\text {betularius }+} \\
& \text { viele wenig viele wenig. }
\end{aligned}
$$

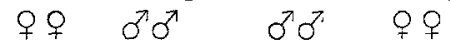

$$
\begin{aligned}
& \underbrace{\text { betularius } \sigma^{7} \times \text { doubledayaria } ᄋ}_{\text {betularius }+ \text { doubledayaria }} \\
& 6 \sigma^{\pi} \quad 2 \text { 웅 }
\end{aligned}
$$

(SMALlWOOD)

Wir haben hier auf diese Dinge nicht einzugehen ${ }^{1}$ ), heben nur hervor, daß in diesen Fällen keine Ausschließlichkeit wie bei GoLDSCHMD'T herrscht und daß in den übrigen Fällen solche „gottgewollte Abhängigkeiten" nicht auszumachen waren, auch nicht bei STEINERT. Ihm brachte ein + doubledayaria, wie oben aus der Zusammenstellnng schon ersichtlich, 75 betularius und zwar $300^{\circ}, 45$, ferner $34 \sigma^{7}$ und 56 ㅇ der Aberration.

1) Vielleicht liegt doch auch bei dem hier neu mitgeteilten Amphidasys-Fall geschlechtsbegrenzte Vererbung vor. In $F_{2}$ sind die $\sigma^{7} \sigma^{7}$ durchweg kleiner wie die $q$. Vergl. darüber Tafel II! Sollte das anf das in $F_{1}$ verwandte $\sigma^{7}$ der Stammform zurückzufübren sein? Wie TafeI II zeigt, ist dies kleiner wie das angekreuzte $Q$ der Aberration. Über diese Dinge hoffe ich später ausführliche und genaue Auskunft geben zu können. 
Schlieblich sei noch anf die von STANDFuss vorzïglich durchgeführte Analyse der Aglia tau und ihrer Aberrationen hingewiesen. Die $a b$. ferenigra und melaina erweisen sich bei Rückkreuzungen mit der Stammform immer als heterozygot. Der Fall ist so bekannt, weil oft erörtert, daß hier nicht weiter darauf eingegangen werden soll. Nun finde ich aber bei ScHRÖDER (1903) ein Zitat, wouach A. WERNER ab. melaina konstant gezogen haben soll. Daran vermag ich in dieser Allgemeinheit einfach nicht zu glauben: denn im Hinblick auf ZIMMERManNs Versuch mit Cymatophora z. B. muß eine Spaltung nach 3:1 erwartet werden. Es wäre das erste und bislang einzige Beispiel einer homozygoten Lepidopteren-Mutante. In diesem Abschnitt konnte aber gerade gezeigt werden, wie allgemein die Heterozygotie melanistischer Mutationen ist.

\section{Die Entstehung der Heterozygotie in melanistischen Mutationen: Additions- nnd Substitutionsmutanten.}

Mit der Feststellung der Heterozrgotic ist die Arbeit noch nicht geleistet, vielmehr ein Problem gestellt. Wie kommt es zur Heterozygotie? In Beantwortung dieser Frage können wir uns zunächst einem Gedankengang von DE VRIES anschließen.

Er fand, daß manche seiner Mutanten bei Kreuzung mit der Stammart diese rein abspalteten. Er ließ deswegen - und das liegt ja sehr nahe - die Mutation in den Geschlechtszellen vor sich gehen. Nun treten oftmals solche "gametische Affektionen" nur in einem einzigen Organismus anf, sagen wir in einem weiblichen. Die Kntwicklung der teilweise affizierten oder veränderten Faktorengesamtheit, des Eies, setzt bei Bisexualität Befruchtung voraus. Die männliche Samenzelle aher bringt ihren ursprünglichen, unceränderten Faktor w mit. Erst die jetzt entstandene Zygote mit den (nehen sehr, sehr vielen andern) Faktoren wS ist entwicklungsfähig, erst daraus entsteht ein äußerlich von der Grundforn abweichender Oroanismus von schwarzer Färbung. Das ist eben nach DE VRIEs der springende Punkt: Die Mutation vollzieht sich in der Stammform, in der Stammform lebt dann gewissermaßen die imaginäre, homozygotische Mutante. Leider ist sie als solche nicht lebensfähig, oder wenigstens findet sich ilrr Dornröschenritter nur selten einmal. Darum ist jede manifestierte Mutante heterozygot. Also:

betularius $\underbrace{\mathrm{W}}_{\mathrm{W}} \times \mathrm{SS}$ betularius (innerlich doubledayaria). 
Demnach ist die neue Form durch das Schicksal der Einzigkeit, das unter ander'n Umständen ein Vorzug wäre, verurteilt zur Bastardnatur, sobald sie in Erscheinung treten will.

Diese Erklärung der Heterozygotie, wie sie sich an DE VRIES anlehnt, ist allerdings nicht die einzig mögliche. GoLDSCHMmT hat wohl zuerst den Gedanken geäußert, diese Aberrationen möchten direkt als heterozygote Mutanten entstanden sein. Wenn wir etwa den von BAUR in seiner "Einführung" mitgeteilten Fall bei Antirrhinum ins Auge fassen, dann scheint fürs erste diese Interpretation gar nicht schwierig zu sein. Aber bei BAUR liegt ein Idealfall vor. Sämtliche Zellen, männliche wie weibliche, sind gleichzeitig mutiert. Dann hat vor allem Selbstbefruchtung statt. Es sei eine weibliche Keimzelle entstanden, die wS konstituiert ist, dazu tritt das gleich beschaffene männliche Element. Der neue Organismus, die Mutante, besteht also aus Zellen wwSS. Der Mechanismus ist unschwer anszudenken, der später eine Verteilung w und $\mathrm{S}$ bewirkt, so daß diese Faktoren je zu 50\% entstehen. Nun denken wir uns den sonst gleichen Fall in das LepidopterenMilieu übertragen. Wie STANDFuss richtig schon bei melaina und ferenigra vermutet, bei dem seltenen Auftreten der Aberration wird wohl immer das $\sigma^{7}$ lediglich $w$ zuführen. Dann kommen wir zu einer Konstitution von wwS für die lebensfähige Mutante. Wie das später zu der bekannten Spaltung w und S führen soll, wie dabei überhaupt die zytologischen Vorgänge sich regulär abspielen sollen, ist mindestens mit unsern jetzigen Vorstellungen von diesen Dingen kaum zu vereinbaren. Es würden sich Hilfsannahmen nötig machen, sei es, daß ww die Fähigkeit zu spalten verliert, sei es, daß ein w irgendwie verloren geht. Auch dieser Punkt müßte bloßer Vermutung überlassen werden.

Das ist ja vorläufig das Leidige solcher Erörterungen, daß wir über die inneren Vorgänge so wenig wissen. Jede Annahme muß in diesem Punkt willkürlich verfahren. Über diese genotypische Seite hinaus liegt aber die Mehrzahl der bis jetzt bekannten Mutationen noch insofern schwieriger wie Antirrhinum, als sie nicht einmal phänotypisch einheitlich sind. So ist bei STANDFUsS unter vielen normalen Geschwistern eine einzelne Mutante aufgetreten. Bei Tower entstanden zu gleicher Zeit decemlineata, pallida und immaculothorax aus Eiern desselben Tieres, obwohl sie allesamt unter gleichen Bedingungen standen. Wenn immer sämtliche Geschlechtszellen affiziert würden, dann ließe sich vielleicht in günstigen Fällen schon nach dem phänotypischen Befund etwas darüber aussagen, ob "Verlust" - oder "Gewinnmutante", dann würden wir mit einem Prinzip der Geschwisterbeurteilung etwas weiter kommen können, 
jedoch kaum bis ans Ende. Letzthin liegen doch die zytologischen Vorgänge, die Gewinn oder Verlust bedeuten sollen, im übrigen dunkel. Demnach kann ich GoLDSCHMIDTs Annahme einer ursprünglichen Heterozygotie schon vor der Befruchtung nicht gelten lassen.

Bleiben wir bei BAURs Idealfall, so würde die dort neu entstandene Sippe mit den merkwürdigen Blüten und deren Anordnung an einem Zweig sowohl phänotypisch wie phylogenetisch etwas völlig Neues bedeuten. BAUR selbst meint, die Mutante sei so eigenartig, daß es ohne Kenntnis ihrer Entstehung schwer sei zu sagen, welcher Familie sie angehöre. Trotzdem soll eine Verlustmutante vorliegen. Ich möchte nur meinen, BAUR setzt hier zwei Erscheinungen überein, die sorglich getrennt werden müssen. Tatsächlich gegeben ist nur die Heterozygotie, die sich in der spätern Spaltung kundgibt. Damit ist über die Entstehùng als Verlust- oder Gewinnmutante gar nichts ausgesagt. Das sind zytologische Vorgänge, die sich unserer Einsicht völlig entziehen. Mit guten Gründen können wir die Wachstums- und Reifeperiode der Geschlechtszellen als die kritische Zeit betrachten. Noch ist keine Reduktion vor sich gegangen. Der Kern führt die Faktoren WW. Mutiert er jetzt zu. Ww, wo w eine gänzlich neue Außeneigenschaft der Anlage nach repräsentiert, wie das oben hervorgehoben wurde, dann soll nach BAUR ein VerIust vorliegen. Aber hier liegt eine Täuschung vor, an der die Schreibweise nach der Presence-Absence-Theorie Schuld trägt. Nichtweiß ist noch lange kein Schwarz. Wenn die Fähigkeit erlischt, eine bestimmte Eigenschaft hervorzurufen, so ist damit noch nicht die andere gegeben, die etwas ganz Neues bedingt. Der Melanismus der Lepidopteren ist nach STANDFuss eine phylogenetische Neuerwerbung. Es ist nicht gut auszudenken, wie dann bereits das Gen dafür in der Erbmasse vorhanden sein soll. Dann wären alle erblichen Abänderungen destruktive Abbauerscheinungen des Organismus. Es genügt, diesen Gedanken ohne Werturteil auszasprechen; denn auf diesem Wege kommen wir zur Metaphysik: zur Annahme einer Vorbildung alles Seienden als Idee in PLATOs Sinne. Rein theoretisch seien die beiden Möglichkeiten erörtert, wie allenfalls eine melanistische Mutante entstehen könnte. Entweder mutiert die homozygote Ovocyte zu WS, oder zu WW addiert sich SS.
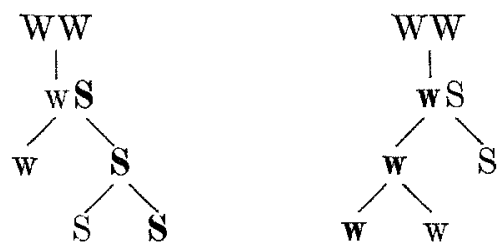
Von einem Verlust kann hier jedenfalls keine Rede sein, wohl aber darf von einer Substitution gesprochen werden. Chemisch ist das ohne weiteres verständlich: diese Umwandlung der substanziellen Einheit eines Faktors in eine von etwas anderem, sagen wir molekularem Aufbau. Weiter möchte ich in den hypothetischen Aussagen nicht gehen und wende mich zur zweiten Möglichkeit.

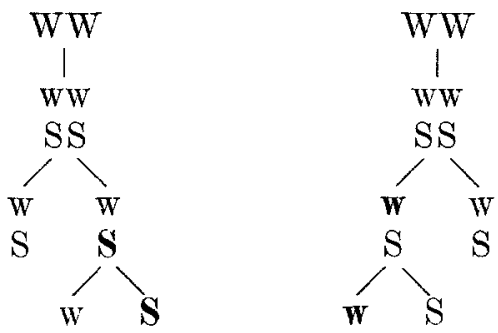

Genau wie vorhin spielen sich die Vorgänge beider Schemen in einem Organismus ab. Welches Gen schließlich durch die Reduktionsteilung entfernt wird, ist rein dem Zufall überlassen. So entstehen von jedem Faktor je 50\% der Gameten. Im Prinzip kommt es hier darauf an, wie sich zu den WW die SS gesellt haben. Das ist wirklich eine Addition an dem unveränderten sonstigen Bestand.

Erinnern wir uns jetzt wieder an BAURs Fall, dann passen auf den offenbar beide Schemen. Es läßt sich eben nach äußerer Inspektion gar nichts entscheiden, ob Substitution oder Addition. Die Schemen stimmen darin überein, daß sie zur Digametie des Organismus führen. Diese Digametie ist freilich nicht das notwendige Ergebnis der ursprünglichen Mutation im einen oder andern Sinne, sondern bedingt durch den von jenem völlig unabhängigen Prozeß der Reduktionsteilung und dem Spiel des Zufalls, das dabei wirkt. Angesichts der regellosen Zahlen, in denen sonst mutierte Zellen auftreten, kann die hälftige Verteilung im Antirrhinum-Fall nicht wohl darauf zurückgeführt werden, daß eben nur die Hälfte affiziert worden sei. Vielmehr ist dieser Endeffekt das Ergebnis jener berührten Teilung und der Wahrscheinlichkeitsrechnung.

Gegenüber diesen Erörterungen ist ein anderer Punkt nebensächlich, muß aber doch geklärt werden. BaURs Mutterpflanze, die also, in der die Mutation vor sich ging, kann nicht als heterozygot bezeichnet werden, wie das der Autor tut. Ein heterozygoter Organismus ist ein solcher, der seinen Ausgang von einer "heterozygoten Zygote" genommen hat. Vielleicht erweist es sich als nötig, hier einen neuen Terminus einzuführen. Ich möchte vorschlagen, von Heterogametie zu sprechen. 
Damit ist nur einer Tatsache Ausdruck gegeben, nicht aber der voransgehenden Ursache. Diese Interpretation behält volle Gültigkeit auch dann, wenn eine vegetative Mutation vorliegt. Selbst dann wird doch der spätere heterogametische Ausgang noch ziemlich spät im individuellen Leben vorbereitet. Demnach erscheint auch bei BauR der Mutterorganismus heterogametisch, und erst die Nachkommen desselben sind wirkliche Heterozygoten. Von diesen Gesichtspunkten aus sind die melanistischen Mutationen völlig parallel zu setzen. Und das kann dureh die Unterschiede, die die Selbstbefruchtung einerseits, andererseits die bloß sporadisch oder aber durchweg erfolgende Mutation schafft, nicht verwischt werden.

Gegenwärtig sind wir außerstande anzugeben, $o b$ in einem bestimmten Falle eine Substitutions- oder Additionsmutante vorliegt. Zugleich stehen wir hier an der Grenze dessen, was das Vererbungsexperiment allein leisten kann. Es muß sich mit dem physiologischen und der Zellforschung verbinden, welch letztere freilich immer noch auf die unbedingt günstigen Objekte wartet.

So gut wir also über das erbtechnische Verhalten der LepidopterenMelanomutationen unterrichtet sind, über ihrer Entstelung liegt auch fernerhin geheimnisvolles Dunkel. Nur soviel scheint mir sicher, daß sie keine Verlustmutanten sind. Darin stimme ich mit GoLDschmidT völlig überein.

\section{Andere Ergebnisse in $\mathbf{F}_{2}$ bei Coleopteren.}

Wie sich oben für die melanistischen Mutationen bei Lepidopteren herausstellte, ergeben sie sämtlich bei Rückkreuzung mit der Ausgangsform eine glatte Spaltung. Wir kennen aber aus andereren Gruppen melanistische Formen, Mutationen natürlich, mit anderem Ergebnis in $F_{2}$. Der erste Fall ist der, daß in $F_{2}$ uur die Mutation anftritt. Das trifft zu für die Kreuzung:

$$
\text { Adalia bipunctata } \times 6-p u s t u l a t a \mathrm{~L} \text {. }
$$

und auch für

$$
\text { Adalia bipunctata } \times 4 \text {-maculata, }
$$

die zuerst von DE MEIJERE, dann von SCHRÖDER mitgeteilt worden

\begin{tabular}{|c|c|}
\hline 3 Kreuzungen & Ad. bipunetata $\sigma^{7} \times 6$-pustulata \\
\hline in 1902 & $\begin{array}{c}24 \text { 우 } 11 \text { o } 6 \text {-pustulata } \\
\text { Ad. bipunctata } \times 6 \text {-pustulata } \sigma^{7}\end{array}$ \\
\hline
\end{tabular}
sind. Der letztere berichtet folgende Experimente: 
26 Kreuzungen

in $1904 / 05$
Ad. bipunctata $\times$ 6-pustulata bez. 4-maculata

$1890^{7}$ ㅇ 6-pustulata bez. 4-maculata.

In $\mathrm{F}_{2}$ ist also vollständige Dominanz der Aberration über die Stammform festzustellen. Gerade das entgegengesetzte Verhalten fand TowER bei Leptirotarsa. Durch gewisse Versuchsbedingungen erzielte er die Form pallida, von deren 89 Individuen $2 \sigma^{7}$ am Leben blieben.

$$
\mathrm{F}_{2} \underbrace{}_{\mathrm{F}_{4}} \underbrace{\frac{2 \delta^{7} \text { pallida } \times \text { decemlineata }}{34 \text { decemlineata }}}_{41 \text { pallida }} \text { 일 pall. }+54 \text { deceml. }
$$

Demnach ist in $F_{2}$ die vollständige Dominanz der Stammform zu konstatieren. Wir haben damit drei verschiedene Fälle kennen gelernt.

$$
\begin{array}{c:c|c}
\text { ww }+\mathrm{wS} & \text { wS } & \mathrm{sW} \\
\text { Amphidasys } & \text { Adalia } & \text { Leptinotarsa }
\end{array}
$$

Für das abweichende Verhalten von Adalia und Leptinotarsa sind die Gründe zu erörtern, zuerst für Leptinotarsa.

Einer Rechtfertigung, warum ich pallida als melanistisch bezeichne trotz der geringen Aufhellung gegenüber decemlineata, bedarf es kaum. Nun sollte man annehmen, daß die pallida gemäß ihrer genau zu verfolgenden Entstehung heterozygot sei. Aber schon TowER und im Anschluß an ihn JoHANNSEN haben das eigentümliche Verhalten der Individuen betont, die aus der Vereinigung einer experimentell mutierten Gamete mit einer normalen entstehen. Die Zygote, aus der pallida hervorgehe, sei "nicht heterozygotisch mutiert", sondern verhalte sich "wie eine aus zwei mutierten Ganeten gebildete Homozygote". Was mit dem durch die Befruchtung eingebrachten Faktor w geschieht, wissen wir nicht. Jedenfalls scheint mir in Anbetracht der vielen pallida, die sogleich entstehen, ziemlich sicher zu sein, daB in der Stammform eine homozygote Substitutionsmutante entstanden ist, also

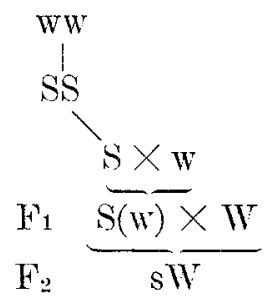

Es geht aber auch nicht an, den eingebrachten Faktor w ohne weiteres "verloren" zu setzen. An diesem Punkte ließe sich die ganzo, 
Willkür dartun, mit der wir jetzt noch infolge unserer Unkenntnis der tieferen Vorgänge interpretieren dürfen. In Perspektion auf die P-Generation ist die Dominanz der Stammform in $\mathrm{F}_{2}$ bemerkenswert. Es könnte an sexuelle Potentialdifferenz gedacht werden; denn der Faktor w $\operatorname{der} \mathrm{P}$ stammt von einem $O$, darüber $\mathrm{F}_{1}$ von einem $\sigma^{\top}$.

So ist ist die manifestierte pallida Substitutionsmutante und homozygot ebenso wie Adalia 6-pustulata. Ob freilich bei letzterer die gleichen Verfahren zur Homozygotie geführt haben, ist nicht sicher. 6-pustulata ist eine sehr alte, daher in sich fixierte Form. Möglich ist es immerhin, daß sie erst in Laufe der Zeit homozygot geworden ist. Diese Entwicklung wäre begünstigt worden durch die unbedingte Überlegenheit des mutierten Faktors über das von bipunctata zugeführte w.

Nach diesen Darlegungen gehören Lymantria, Aglia, Cymatophora, Amphidasys in eine Kategorie eng zusammen. Was sie zusammenfügt, ist sehr viel: die Heterozygotie der manifestierten Mutante. Bei der Seltenheit, mit der bei allen angeführten Lepidopteren die Aberration auftritt, wird in der großen Mehrzahl der Fälle, vielleicht sogar ausschließlich, Befruchtung durch die Stammform statthaben. Dann müssen natïrlich heterozygote Melanismen auftreten.

Dieser Kategorie treten Adalia und Leptinotarsa gegenüber als homozygote Formen. Getrennt sind sie durch eine Kleinigkeit: dort die Dominanz, hier die Rezessivität des mutierten Faktors in $\mathrm{F}_{2}$. Vererbungstheoretisch hat das wenig zu bedeuten; denn beide unterliegen genau so wie die besprochenen Lepidopteren-Melanomutanten der alternativen Vererbung. ScHRöDER will die „völlige Prävalenz nigristischer progressiver Aberrationes" nur aus konstitutionellen Ursachen erklären. „Ob Mutation oder fluktuierende Variabilität vorliegt, bedingt nicht die Konstanz einer Form, sondern immanente, öfters nicht gesetzmäßig ausdrückbare, im artlichen Organismus begründete Eigentümlichkeiten." Dieser mythologischen Auffassung gegenüber weisen wir noch einmal besonders auf den streng alternativen Verlauf dieser Prozesse hin. Es hat sich noch immer als angebracht erwiesen, nicht neue Hypothesen zu setzen, sondern auf dem gesicherten Boden des Mendelismus weiter zu bauen.

\section{Zusammenfassung.}

1. Melanistische Formen treten auf in allen Ausprägungen von geringem schwarzen Einschlag bis zur höchsten Potenzierung. 
2. Vererbungstheoretisch haben die Melanismen die Bedeutung entweder von bloßen Modifikationen oder aber von Mutationen.

3. Aus einer Population z. B. von Lymantria lassen sich reine Linien isolieren.

4. Umgekehrt beruht die Variabilität einer solchen Population auf der Kombination von Faktoren, die durch je eine reine Linie repräsentiert sind.

5. Es ist nicht schwer auszudenken, daß die ganze Mannigfaltigkeit die Wirkung weniger mendelnder Faktoren bez. reiner Linien ist.

6. Meistens sind die Individuen einer solchen Linie ideal binomial aufgeteilt.

7. Besonders bei T-Experimenten treten asymmetrische Kurven auf, die doch wohl dartun, daß der Organismus nicht den äußeren Bedingungen parallel reagiert, sondern daß sich seine Modifikabilität innerhalb gewisser Grenzen bewegt.

8. Überhaupt erweist sich das physiologische T-Experiment zur Ergänzung des Vererbungsexperiments direkt als notwendig, da es aus dem Organismus die volle Reaktionsnorm herausholt. Vielleicht hat dieser Satz recht allgemeine Geltung.

9. Die reinen Linien sind transgressiv variabel, überschneiden sich also gegenseitig.

10. Phänotypisch völlig schwarze Formen können bis zu weiß persönlich fluktuieren (GotoschMIDT). Das Vermögen weißer Individuen kennen wir so genau noch nicht.

11. Durch Kreuzung reiner Linien ergeben sich zweigipflige Kurven.

12. Selektion extremer Abweicher erbrachte keinerlei Erfolg.

13. Eine melanistische Mutation ist äul3erlich von einer bloßen Modifikation nicht zu unterscheiden, wie überhaupt einer Form der genotypische Charakter nicht von außen angesehen werden kann. Es gibt Melanomutanten, die durchaus nicht typisch, d. h. rein schwarz sind. Vergl. die Aberrationen von Aglia tau!

14. Alle melanistischen Mutanten bei Lepidopteren sind heterozygot.

15. Die Ursache der Heterozygotie liegt in der Befruchtung durch eine unveränderte Gamete. Damit ist zugleich die Tatsache illustriert, daß genotypische Affektionen weder gleichmäBig den einzelnen Organismus, noch auch die betreffende Gruppe von Individuen betreffen.

16. Die bis jetzt experimentell untersuchten Mutanten bei Coleopteren sind homozygot. 
17. Ob Adalia und Leptinotarsa hinsichtlich der Ursache ihrer Homozygotie analog zu setzen sind, läßt sich zurzeit nicht entscheiden.

18. Wenn auch nicht dargetan werden kann, daß die Melanomutanten bei Lepidopteren und Coleopteren Gewinnmutanten im bisherigen Sinne dieses Wortes sind, so kann doch eine etwaige Deutung als Verlustmutanten sicher ausgeschlossen werden. Das ist bemerkenswert, da BAUR (1911) kein Beispiel für additive Mutationen geben konnte.

19. Sekundär müssen bei Gewinnmutationen substitutive und eigentlich additive auseinandergehalten werden. Der substitutive Charakter von Leptinotarsa ist wahrscheinlich gemacht.

20. In welche Kategorie die Lepidopteren gehören, wissen wir noch nicht. Die Entscheidung darüber kann unter einer gewissen Voraussetzung das Prinzip der Geschwisterbeurteilung, d. h. das Zuchtexperiment im Verein mit der Zellforschung bringen.

21. Bei Lepidopteren und bei Adalia ist die melanistische Mutation der Stammform gegenüber dominant. Diese unbedingte Prävalenz ist ein Zeichen für den progressiven Charakter.

22. Für alle melanistischen Mutationen - ohne Rücksicht auf Genese, gametische Konstitution und Valenz - gilt streng die alternative Vererbung. Wäbrend noch Gross hier einen Fall sui generis konstruieren will, reiht sich uns die Erscheinung glatt in den Zusammenhang der mendelistischen Phänomene ein. Bei der meist erfolgenden Paarung der Mutante mit der Stammform erfolgt eine Spaltung nach dem Rückkreuzungsschema: Das hat sich zuerst durch STANDFuss' Experimente mit Aglia tau ergeben.

\section{Literatur.}

GoLDschumт: Einführung in die Vererbungswissenschaft. Leipzig 1911.

- Erblichkeitsstudien an Schmetterlingen I. Diese Z. Bd. VII, 1912.

GRoss: Über einige Beziehungen zwischen Vererbung und Variation. Biol. Zentrbl. 26, 1906 .

- Über Vererbung und Artbildung. Ebenda 31, 1911.

Hasebroek: Eine bemerkenswerte bei Hamburg auftretende Schmetterlingsmutation. Umschau 1913 , S. $1020 / 22$.

Johannsen: Elemente der exakten Erblichkeitslehre. II. Aufl. Jena 1913.

DE MeIJERx: Zur Vererbnng des Geschlechts und der sekundären Geschlechtsmerkmale. Arch. f. Rass. Gesbiol. 1913, S. 1-36.

SchröDer: Abraxas. Allg. Z. f. Entomologie. Bd. VIII, 1903.

- Adalia. Z. f. wiss. Insektenbiologie V, 1909, S. 132-134. 
Schrö̀er: Lymantria. Ebenda IV, 1908.

- Zur konstitutionellen Prävalenz der Melanismen. Ebenda V, 1909, S. 27-29.

STANDFuss: Handbuch 1896.

- Die Resultate 30 jähriger Experimente mit Bezug auf Artenbildung und Ungestaltung in der Tierwelt. Verh. der Schweiz. Naturf. Ges. 88, 1905.

- Die alternative oder diskontinuierliche Vererbung und ihre Veranschanlichung an den Ergebnissen von Zuchtexperimenten mit Aglia tau und deren Mutationen. Deutsche Entom. Nationalbibliothek I. 1910.

Stickfu: Über Melanismus und Nigrismus bei Lepidopteren. Z. f. wiss. Insektenbiol. VII, 1911.

Tower: An Investigation of Evolution in Chrysomelid Beetles of the Genus Leptinotarsa. Carnegie Institution Publications Washington 48, 1906.

- The Determination of dominance and the Modification of Belavior in alternativ (Mendelian) Inheritance, by conditions surrounding or incident upon the Germcells at fertilization. Biol. Bull. 18, 1910.

\section{Erläuterungen $z u$ den Tafeln.}

Tafel I. Aus einer Population der Nonne ausgewählte Individuen, im Jahre 1907 in der Umgebung von Leipzig gefangen, links $\delta$, rechts $O$. Auch die beiden dunkelsten Stïcke lassen noch einzelne Zeichnungselemente erkennen.

Tafol II. Birkenspanner, Amphidasys betularius L. Oben das elterliche Paar, rechts $a b$. doubledayaria, 1910 in Kopulation bei Leipzig gefunden. Die übrigen Tiere $\mathrm{F}_{2}=$ Nachkommen. Zunächst 6 weiße Tiere mit individueller Variation der Zeichnungselemente: das Schwarz nimmt $z u$ und bildet schließlich zusammenhängende Zickzackbänder, doch liegt diese Variationsreihe duxchaus innerhalb von Weiß. 5. Reihe: schwarze $\mathrm{F}_{2}$, größtes und kleinstes Tier, derselbe Gegensatz in Weiß, s. 6. Reihe! Die kleinen Tiere $\sigma^{\top}$. Sämtliche abgebildeten Tiere mit Ausnahme der ersten Reihe gespannt sofort nach dem Ausschlüpfen. 


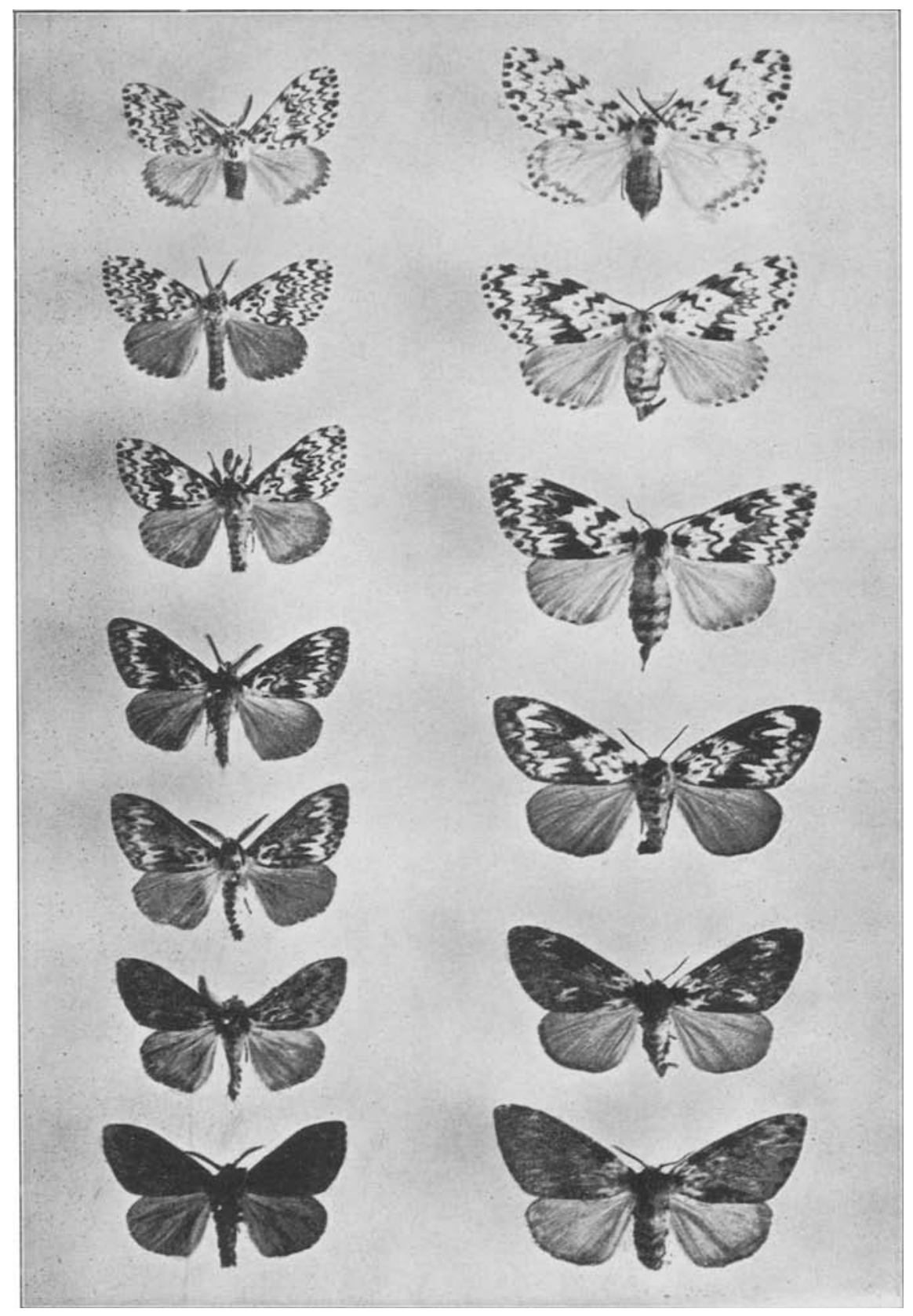

Gerschler: Lymantria monacha 


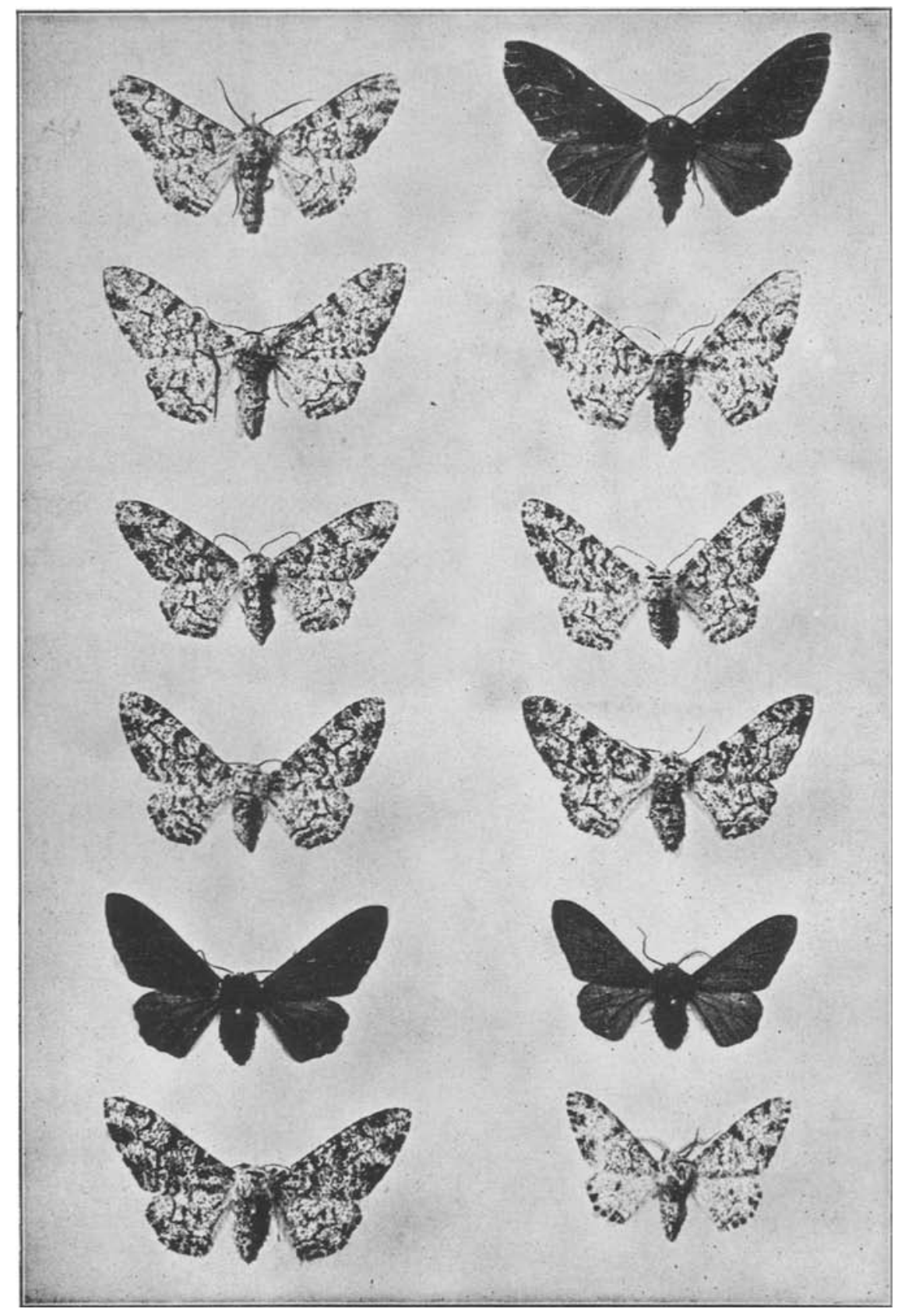

Gerschler: Amphidasys 